\title{
Quantitative nuclear magnetic resonance imaging: characterisation of experimental cerebral oedema
}

\author{
D BARNES, W I MCDONALD, G JOHNSON, P S TOFTS, D N LANDON \\ From the Department of Clinical Neurology, Institute of Neurology, Queen Square, London UK
}

SUMMARY Magnetic resonance imaging (MRI) has been used quantitatively to define the characteristics of two different models of experimental cerebral oedema in cats: vasogenic oedema produced by cortical freezing and cytotoxic oedema induced by triethyl tin. The MRI results have been correlated with the ultrastructural changes. The images accurately delineated the anatomical extent of the oedema in the two lesions, but did not otherwise discriminate between them. The patterns of measured increase in $T_{1}{ }^{\prime}$ and $T_{2}{ }^{\prime}$ were, however, characteristic for each type of oedema, and reflected the protein content. The magnetisation decay characteristics of both normal and oedematous white matter were monoexponential for $T_{1}$ but biexponential for $T_{2}$ decay. The relative sizes of the two component exponentials of the latter corresponded with the physical sizes of the major tissue water compartments. Quantitative MRI data can provide reliable information about the physico-chemical environment of tissue water in normal and oedematous cerebral tissue, and are useful for distinguishing between acute and chronic lesions in multiple sclerosis.

Magnetic resonance imaging (MRI) is of proven value for the detection of abnormalities in the brain in a variety of diseases of the central nervous system. Despite its sensitivity to pathological changes, however, the abnormal images do not provide specific information about the nature of the underlying pathology. ${ }^{1}$

In addition to producing images which demonstrate qualitative changes, MRI can also provide quantitative data about a tissue. The relaxation times, $T_{1}$ and $T_{2}$ and magnetisation decay characteristics are of particular interest as they reflect not only the amount of water in a tissue but also its physicochemical environment. ${ }^{2}$ These facts raise the possibility that specific pathological changes in a tissue might be characterised in terms of the resultant changes in its nuclear magnetic resonance (NMR) properties. We have investigated this possibility by studying experimental models of cerebral oedema in vivo using quantitative MRI techniques; the NMR characteristics have been compared with the morphological features of the lesions. Two models of cerebral oedema have been studied in cats. First, a

Address for reprint requests: Prof WI McDonald, Institute of Neurology, Queen St, London WCIN 3BG, UK.

Received 24 April 1986.

Accepted 25 May 1986 cortical freezing lesion has been used to produce vasogenic cerebral oedema in the underlying white matter. The lesion is characterised by expansion of the extracellular space which contains protein-rich fluid as a result of damage to the blood vessels. ${ }^{3}$

The second model is cytotoxic oedema induced by the systemic administration of triethyl tin (TET) sulphate. This type of oedema is characterised by splitting of the myelin sheaths at the intraperiod line followed by progressive accumulation of oedema fluid between the separated lamellae to form intramyelinic vacuoles. ${ }^{4}$ Unlike vasogenic oedema, the oedema fluid in this form of cytotoxic oedema lacks a significant protein content. ${ }^{5}$

In a previous paper ${ }^{6}$ we described in detail the correlation between the NMR and morphological features of cytotoxic oedema. In this study we have compared the findings in vasogenic and cytotoxic cerebral oedema in order to determine whether it is possible to gain an insight into the nature of pathological changes in tissue water using quantitative MRI.

\section{Methods}

Twenty-six adult cats (weighing $2-3.5 \mathrm{~kg}$ ) were used in these experiments. Vasogenic oedema was induced by means of a cold lesion in 16 animals. The cold lesion was produced by the following method: general anaesthesia was induced using pentobarbitone sodium $(40 \mathrm{mg} / \mathrm{kg}$ body weight $)$ by intra- 
peritoneal injection, and the animal was positioned in a stereotactic head frame. A burr hole was then made $18-20 \mathrm{~mm}$ in front of the external auditory meatus and $8-10 \mathrm{~mm}$ to the right of the midline and the exposed dura protected by a piece of thin plastic film. A circular brass chuck, $6 \mathrm{~mm}$ in diameter, was cooled in liquid nitrogen and applied to the dural surface for 25 seconds. After removal of the chuck, the frozen cortex was allowed to thaw, the plastic film removed and the soft tissues sutured in layers.

Cytotoxic oedema was induced in 10 animals by the intraperitoneal injection of one to four doses of TET sulphate ( $1 \mathrm{mg} / \mathrm{kg}$ body weight).

\section{Imaging}

NMR imaging was performed on a Picker International system operating at 0.5 Tesla. A home-made saddle-shaped receiver coil $10 \mathrm{~cm}$ in both diameter and length was used, and the imaging sequences were modified to produce a $15 \mathrm{~cm}$ field of view. The reconstruction method used was 2-dimensional Fourier transformation. The pixel dimensions were $0.6 \times 1.2 \mathrm{~mm}$ and the slice thickness, $5 \mathrm{~mm}$.

Of the 16 animals in which cold lesions were made, all were scanned at 24 hours, and six were re-examined at 72 hours after the operation. Those with cytotoxic oedema were examined at intervals up to 4 days after the first dose of TET sulphate. Each imaging session lasted approximately 3 hours during which a series of spin-echo (SE) ( $T_{e}$ from 40 to $1120 \mathrm{~ms}$ ) and inversion-recovery (IR ( $T_{i}$ from 100 to $700 \mathrm{~ms}$ ) images ${ }^{7}$ were acquired in the coronal plane. This plane was found to be the most useful since it provided the best anatomical detail, and the depth of abnormal tissue relative to the slice thickness of $5 \mathrm{~mm}$ minimised partial volume effects.

When imaging the cold lesions, the antero-posterior extent of the underlying oedema was initially ascertained using sagittal views to enable the series of coronal images to be centred on the oedematous white matter. The cytotoxic oedema, which involved the cerebral white matter diffusely, was imaged at the same level to minimise the effects of regional variations in the NMR characteristics of white matter when comparing the two lesions.

The signal intensity of all imaging sequences depends to varying extents upon protein density, $T_{1}$ and $T_{2}$. Thus SE images are particularly sensitive to differences in $T_{2}$ between tissues, whereas IR images depend more on differences in $T_{1}$ for image contrast. The SE sequences were modified by the addition of an extra $90^{\circ}$ pulse after the echo which completely saturated the magnetisation. This resulted in an equal $T_{1}$-dependence of all $S E$ regardless of $T_{e}{ }^{6}$.

The relaxation times were read directly from calculated images produced from a two-point method by computer algorithms: the $T_{1}$ from an SE (40/2000) and an IR $(700 / 2000 / 40)$, and the $T_{2}$ from two $S E(40 / 2000$ and $120 / 2000$ ) images. The values for $T_{1}$ and $T_{2}$ obtained in this way must be regarded as approximations to the true relaxation behaviour of the tissue for reasons which are dealt with later, and will therefore be referred to as $T_{1}{ }^{\prime}$ and $T_{2}{ }^{\prime}$. In addition to the relaxation times, the magnetisation decay characteristics of the abnormal tissue for both $T_{1}$ and $T_{2}$ relaxation were determined by measuring the signal intensity of the region of interest from the screen and fitting the data to the best mono-, bi- and triexponential decay functions using a computed least-squares fitting procedure. An F-test was then applied to determine the most appropriate function.

\section{Determination of water content}

Three animals with vasogenic and three with cytotoxic oedema were killed after the final imaging session by an overdose of anaesthetic following which the brains were removed and sectioned. Samples of tissue $(0.05-0.1 \mathrm{mls})$ were taken from the base of the oedematous gyrus for wetdry weight determination of water content. They were dried in an oven at $60^{\circ} \mathrm{C}$ until their weights had been stable for 48 hours.

\section{Electron microscopy}

Two animals with cytotoxic and six with vasogenic oedema were killed by perfusion-fixation through the ascending aorta via the left ventricle with $3 \%$ glutaraldehyde in $0.1 \mathrm{M}$ cacodylate buffer at a pH of $7 \cdot 4.1500 \mathrm{mls}$ of fixative were perfused over a period of 15 minutes at an effective pressure of $180 \mathrm{~mm} \mathrm{Hg}$. The brains were removed after a further 48 hours in fixative and tissue samples taken from the regions indicated in fig 1 . They were post-fixed in $1 \%$ osmium tetroxide, dehydrated in graded ethanol solutions and embedded in epoxy resin for microscopy. Semi-thin sections ( 0.5 microns) were stained with toluidine blue for light microscopy and ultra-thin sections $(60 \mathrm{~nm})$ with methanolic uranyl acetate and Reynold's lead citrate for transmission electron microscopy.
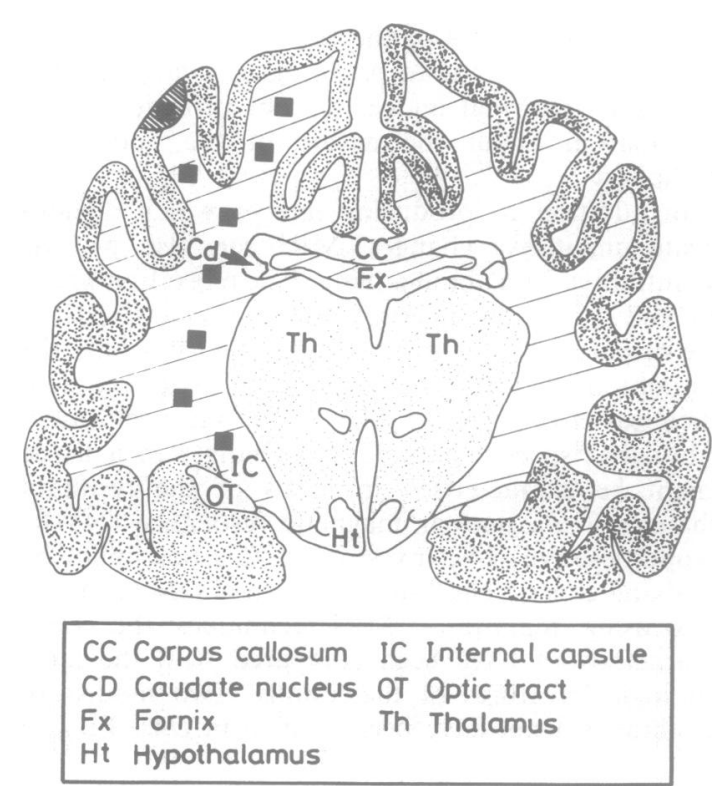

Fig 1 Schematic coronal section of the cat brain showing the site of application of the cold probe and approximate sites from which tissue sample were taken for electron microscopy (回. 


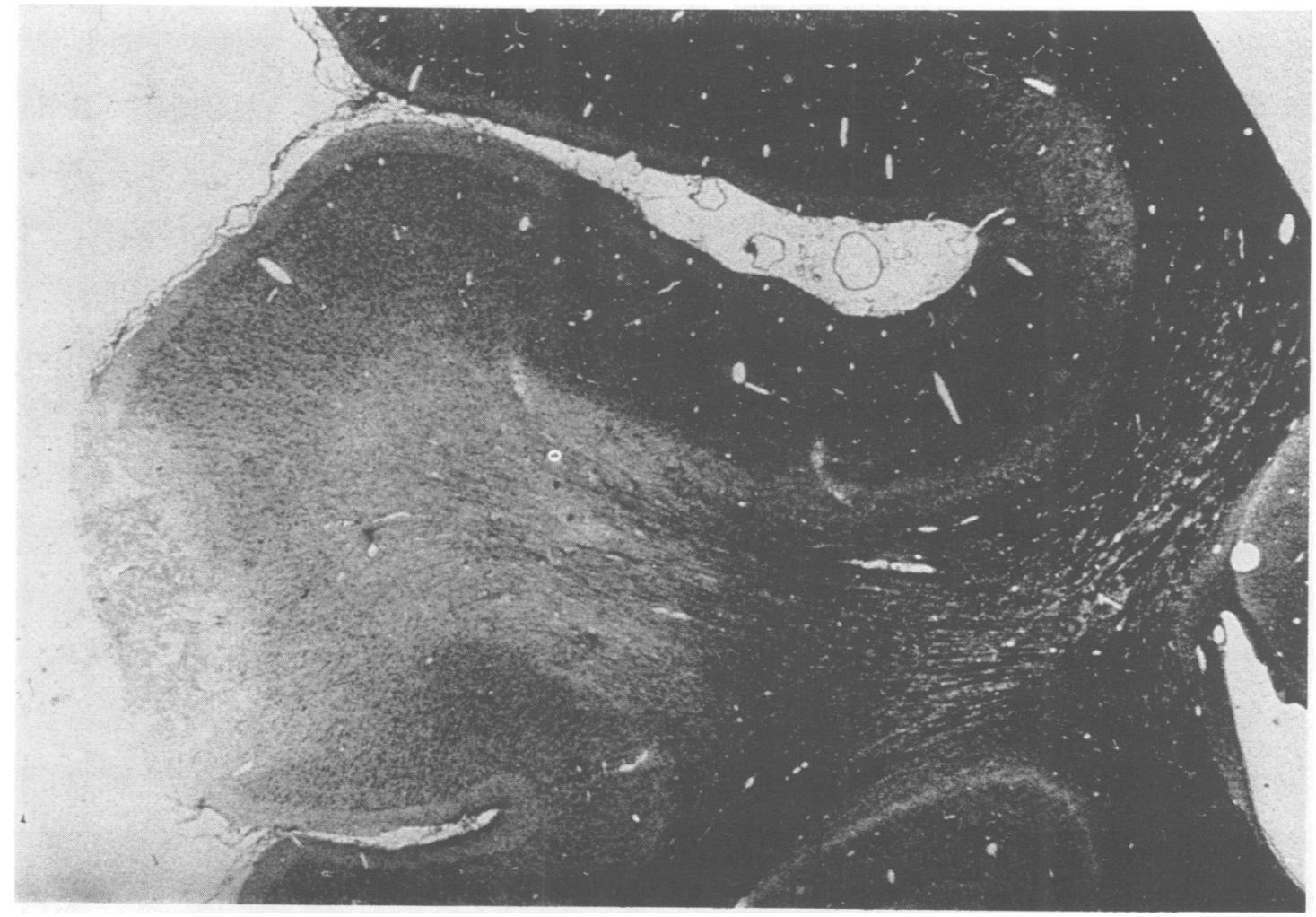

Fig 2 Light micrograph of the suprasylvian gyrus showing the localised superficial cortical lesion and widening and pallor of the subjacent white matter (Luxol Fast Blue-Cresyl Fast Violet; $\times 13$ ).

\section{Light microscopy}

One animal with cytotoxic, and one with vasogenic oedema were perfused with formol-saline to provide paraffin sections for light microscopy.

\section{Results}

Although every attempt was made to standardise the method of production of the cold lesions throughout the series of experiments, the nature and severity of the pathological changes seen at different times were variable. Similarly, animals showed variable susceptibility to TET sulphate but the dosage regimes were adjusted to produce comparable abnormalities on the NMR images.

\section{Microscopic appearances}

Vasogenic oedema. A superficial necrotic cortical lesion marked the site of application of the cold probe. The subjacent suprasylvian gyrus was widened and showed marked pallor when compared with the normal side (fig 2). The changes were maximal immediately subjacent to the cortical lesion, gradually becoming less marked in the deeper white matter. The lateral gyrus and internal capsule were involved to a lesser extent, as was the ipsilateral side of the corpus callosum, although the oedema did not appear to

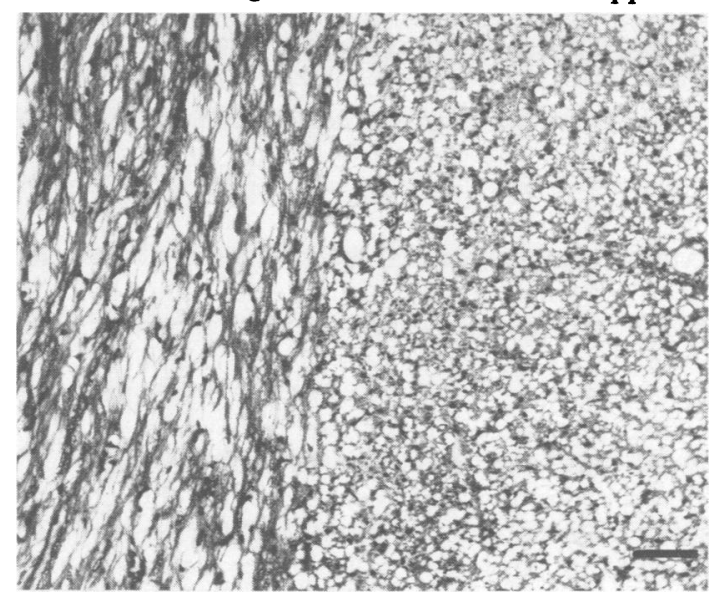

Fig 3 Light micrograph of white matter affected by cytotoxic oedema showing the spongy appearance imparted by the intramyelinic vacuoles (Toluidine Blue;

Bar $=100 \mu \mathrm{m}$ ). 

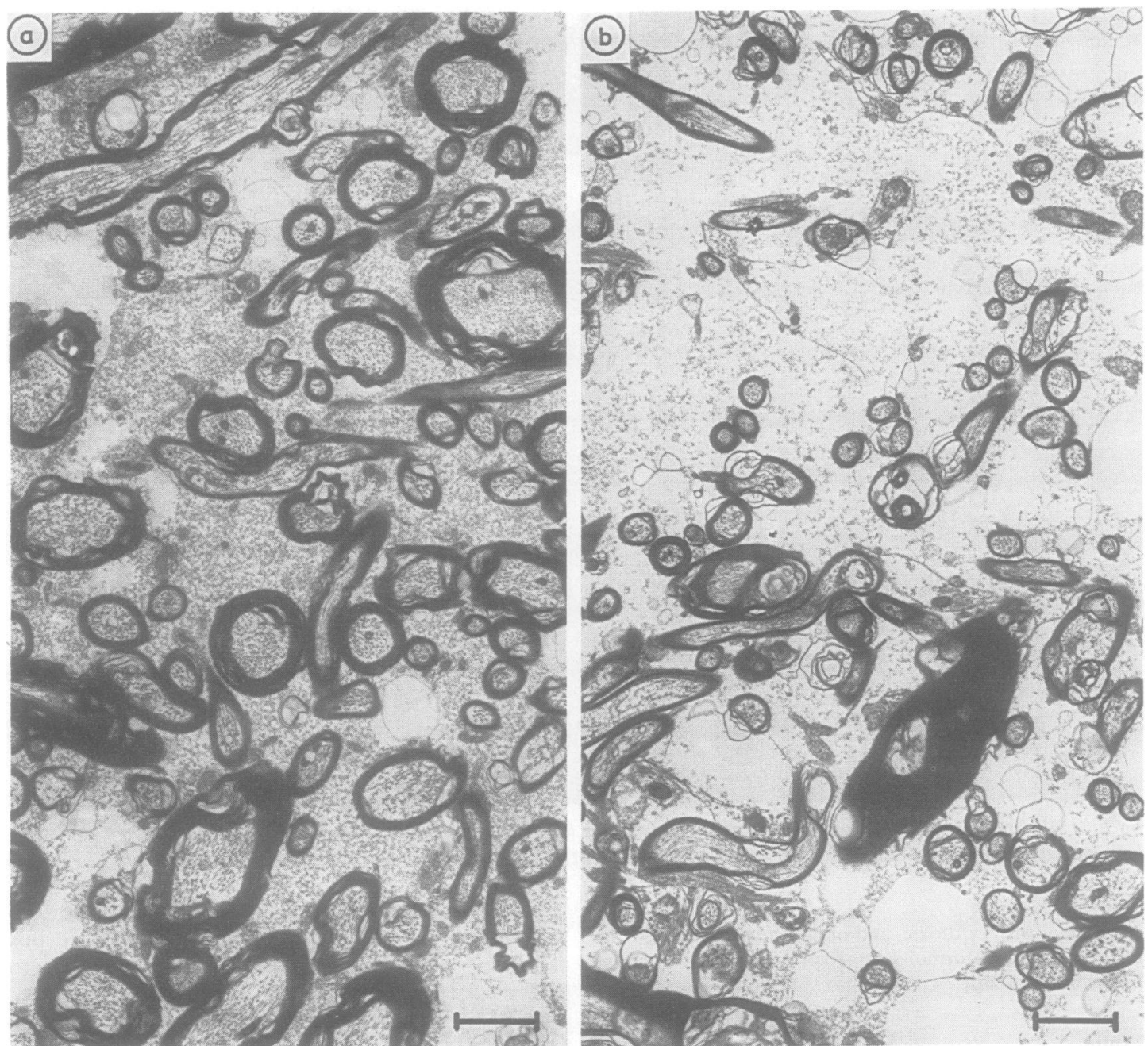

Fig 4 Electron micrographs of vasogenic oedema at (a) 1 day and (b) 3 days following the cold lesion. The extracellular space is enlarged and contains extravasated plasma protein (Bars $=1 \mu \mathrm{m})$.

cross the midline.

At higher magnification, the myelinated fibres were seen to be widely separated from each other in contrast to the normal compact configuration. The appearances were identical at 24 and 72 hours.

Cytotoxic oedema The cerebral white matter showed diffuse pallor compared with normal; that within the gyri, the corpus callosum and the internal capsule adjacent to the basal ganglia being most abnormal in appearance. At higher magnification, the predominant abnormality was a spongiform appearance imparted to the white matter by the presence of numerous vacuoles (fig 3 ). Individual vacuoles appeared to follow the direction of the myelinated fibres, those running longitudinally appearing cigarshaped, and those seen in transverse section, circular.

\section{Electron microscopy}

Vasogenic oedema Three lesions were examined at 1 day and three at 3 days following the production of the cold lesion. In each lesion, the oedematous process was characterised by expansion of the extracellular space in the involved white matter resulting in abnormal separation of the nerve fibres and glial elements (fig 4). The changes were most marked in the suprasylvian gyrus subjacent to the site of application of the cold probe, and appeared progressively less severe in samples from the lateral gyrus and internal capsule, more remote from. it. The size of the extra- 


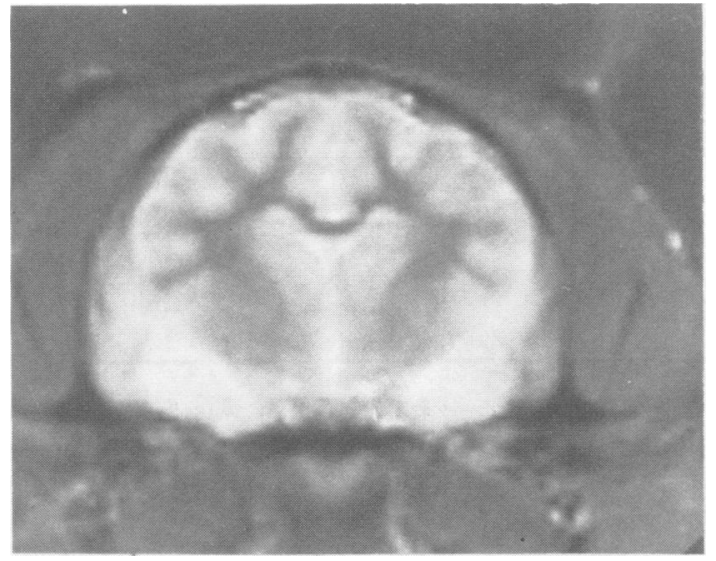

Fig $5 M R I$ (coronal section) of a control animal showing the normal distribution of grey and white matter

(Inversion-recovery; $T_{\mathrm{i}}=200 \mathrm{~ms}, T_{\mathrm{r}}=2000 \mathrm{~ms}$ ) (see fig 1 ).

cellular space in the oedematous white matter was generally greater at day 1 than at day 3 . The oedema did not affect the contralateral hemisphere.

The expanded extracellular space contained fluffy electron-dense material with an appearance typical of plasma protein. This material was of variable density in different regions of individual lesions, but was always much more dense at 1 day than at 3 days after induction of the lesion (fig $4 a$ and $b$ ). The astrocytic processes in the oedematous region appeared swollen at both time intervals, but more so on day 3 than on day 1 . The oligodendrocytes and myelinated nerve fibres appeared normal.

Cytotoxic oedema As previously described, ${ }^{6}$ the predominant abnormality was severe disruption of the myelin sheaths. The myelin lamellae were separated at the intraperiod line and the spaces between them were expanded to form vacuoles which often exceeded $15 \mu \mathrm{m}$ in diameter. The contents of the vacuoles were devoid of electron-dense material in contrast to the oedema spaces in vasogenic oedema. The extracellular space was not visibly enlarged, and the astrocytic processes appeared normal.

\section{MRI appearances}

A coronal image taken from a healthy control is shown in fig 5 in which the normal pattern of white matter can be seen.

Vasogenic oedema The quantitative NMR data from the cold lesions was taken from the base of the suprasylvian gyrus in each experiment. The appearances of vasogenic oedema are shown in fig 6.

Although abnormal signal enhancement was visible in the cortex at the site of application of the cold probe, the remainder of the grey matter appeared

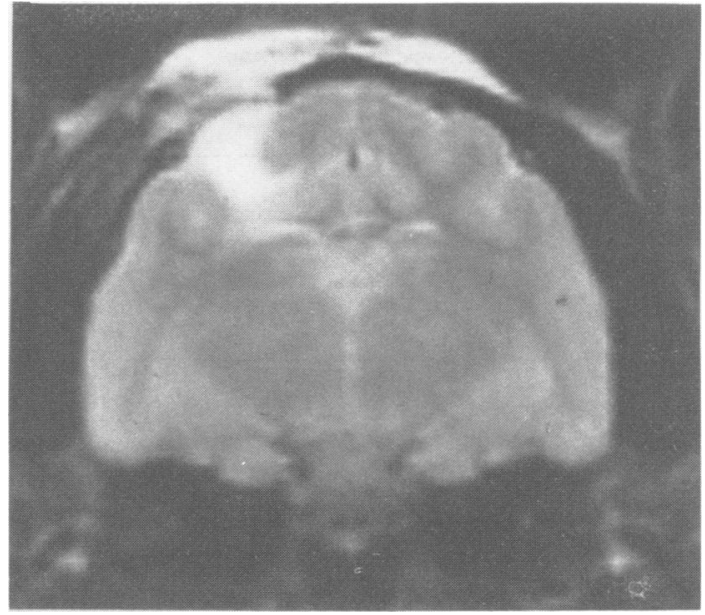

Fig 6 MRI taken 24 hours after the cold lesion. The right suprasylvian gyrus is widened and shows marked signal enhancement which extends into the the internal capsule but does not cross the midline (Spin-echo; $T_{\mathrm{e}}=80 \mathrm{~ms}$, $T_{\mathrm{r}}=2300 \mathrm{~ms}$ ).

unaffected. The most abnormal signal was seen in the underlying white matter, its anatomical distribution conforming to previous descriptions. ${ }^{8}$ It was possible to identify the region of the cortical lesion itself and to delineate accurately the extent of the oedematous white matter as determined histologically. Whereas in the IR images it was not possible to distinguish between the superficial necrotic lesion and underlying vasogenic oedema, the SE images not only differentiated between these, but also provided more accurate information about the extent and severity of the oedema.

Cytotoxic oedema The image appearance of this le-

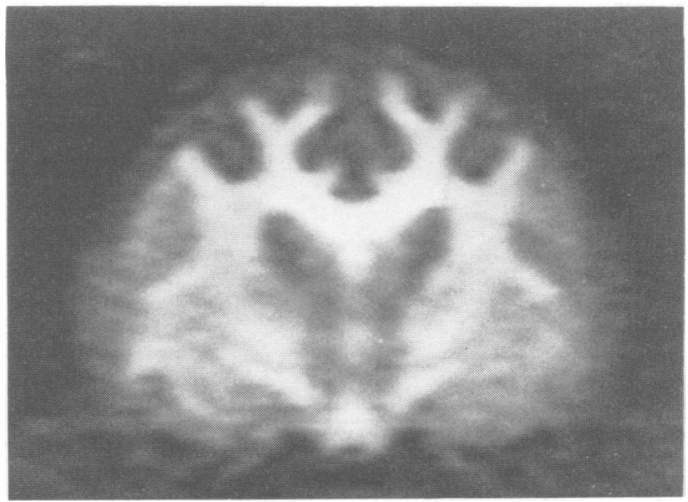

Fig 7 MRI demonstrating diffuse signal enhancement from the cerebral white matter due to severe cytotoxic oedema (Spin-echo; $T_{\mathrm{e}}=240 \mathrm{~ms}, T_{\mathrm{r}}=2000 \mathrm{~ms}$ ). 
Table 1 Absolute values for $T_{1}^{\prime}$ and $T_{2}^{\prime}$ for normal and oedematous white matter and the percentage increase and ratio between them for each experimental group

\begin{tabular}{|c|c|c|c|c|c|}
\hline Experimental group & $T_{1}(m s)$ & $T_{2}(m s)$ & $\% T_{1}$ & $\% T_{2}$ & $\% T_{1} / T_{2}$ \\
\hline $\begin{array}{l}1 \text { day cold }(n=16) \\
3 \text { day cold }(n=6) \\
\text { TET }(n=10) \\
\text { Control }(n=36)\end{array}$ & $\begin{array}{l}887 \pm 128 \\
(715-1160) \\
770 \pm 54 \\
(699-860) \\
575 \pm 46 \\
(510-640) \\
476 \pm 10\end{array}$ & $\begin{array}{l}172 \pm 37 \\
(118-265) \\
159 \pm 16 \\
(142-184) \\
115 \pm 12 \\
(97-130) \\
81 \pm 4\end{array}$ & $\begin{array}{l}87 \pm 21 \\
(50-150) \\
58 \pm 13 \\
(46-81) \\
27 \pm 7 \\
(11-33)\end{array}$ & $\begin{array}{l}103 \pm 31 \\
(48-200) \\
87 \pm 17 \\
(72-123) \\
52 \pm 12 \\
(24-63)\end{array}$ & $\begin{array}{l}0.87 \pm 1.4 \\
(0.72-1.15) \\
0.67 \pm 0.04 \\
(0.64-0.73) \\
0.46 \pm 0.07 \\
(0.36-0.59)\end{array}$ \\
\hline
\end{tabular}

sion has been described in detail previously. ${ }^{6}$ The signal from the cerebral white matter was enhanced diffusely (fig 7), but in keeping with the histological changes, the degree of enhancement was greatest in the gyri and the white matter adjacent to the basal ganglia.

It was not possible to distinguish between regions of cytotoxic and vasogenic oedema of comparable severity on the basis of their image appearances alone.

\section{Relaxation times}

Table 1 summarises the relaxation data from both the normal and experimental groups of animals.

We have considered the relaxation times obtained from experimental animals in terms of percentage increases above their pre-morbid values in order to facilitate comparison between individual animals with different normal values. Within each group the relative proportional increases in the relaxation times remain constant regardless of oedema severity. In 1 day vasogenic oedema the relaxation times were increased in approximately the same proportion for all degrees of severity, whereas in 3 day vasogenic oedema, $\mathrm{T}_{2}{ }^{\prime}$ was increased by approximately $50 \%$ more than $\mathrm{T}_{1}{ }_{1}^{\prime}$. Cytotoxic oedema, at all stages of development

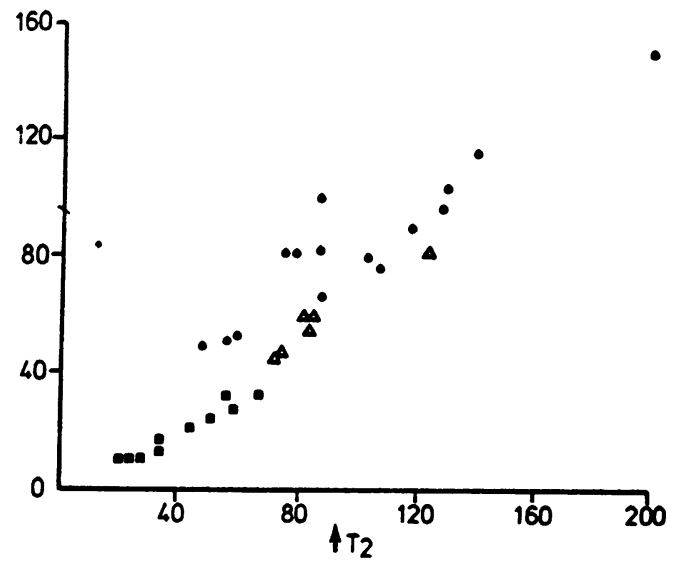

Fig 8 Graph showing \% increase in $T_{1}{ }^{\prime}$ versus $T_{2}{ }^{\prime}$ for each animal $(\square=$ cytotoxic; $=$ vasogenic at 1 day; and $\Delta=$ vasogenic at 3 days). showed an increase in $T_{1}{ }^{\prime}$ which was at least twice that in $T_{1}{ }^{\prime}$. The difference in the ratio of percentage increases in $T_{1}{ }^{\prime}$ to $T_{2}{ }^{\prime}$ between 1 and 3 day vasogenic $(\mathrm{p}<0.001)$, and between 3 day vasogenic and cytotoxic oedema $(p<0.01)$ were significant using the Mann-Whitney test for non-parametric, unpaired data. The relaxation data for individual animals are given in fig 8 .

Magnetisation decay characteristics

The magnetisation decay characteristics of a tissue might be expected to provide information about sepa-

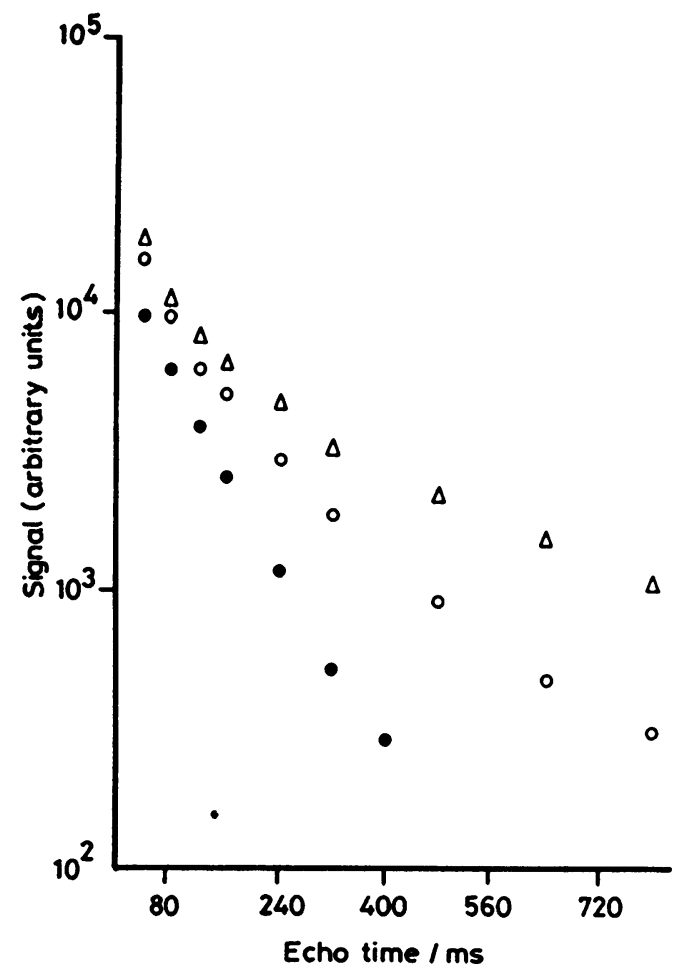

Fig 9 Graph showing the progressive changes in the magnetisation decay characteristics of white matter during the development of cytotoxic oedema $(O=$ control, $O=2$ days and $\triangle=4$ days after the first dose of TET sulphate). 
rate microscopic tissue water compartments. In the present experiments the $T_{1}$ magnetisation decay was always found to be monoexponential, whereas in $5 / 6$ normal animals and all those with oedematous white matter produced non-exponential $T_{2}$ magnetisation decay curves. An example is shown in fig 9.

When the curve-fitting procedure was applied, the addition of a third exponential function did not significantly improve the fit in any experiment and therefore all magnetisation decay curves were treated as biexponential, comprising a short- $\mathrm{T}_{2}$ and a long$\mathrm{T}_{2}$ component. The relaxation times derived from the significantly biexponential decay functions for both components and the percentage of the observed signal derived from the long- $\mathrm{T}_{2}$ component are shown in table 2.

There were no significant differences between any of the groups in the relaxation time of their short- $\mathrm{T}_{2}$ components, whereas that of the long- $T_{2}$ component was considerably greater in each experimental group than in healthy controls. The relative amplitude of this component, which comprised about $21 \%$ of the signal in the control group, was increased to $55-65 \%$ in vasogenic oedema.

\section{Water content}

The water content of normal and oedematous white matter, as determined by estimation of wet and dry weights, are shown in table 3.

The mean increment in water content of tissue affected by cytotoxic oedema was $6 \cdot 3 \%$ and by vasogenic oedema $12 \cdot 3 \%$.

\section{Discussion}

The morphological characteristics of vasogenic oedema reported in these experiments conform to previous descriptions. ${ }^{910}$ The time course and severity of the oedema, however, showed marked variability despite the standardised method of production of the cold lesions. The degree of expansion of the extracellular space was generally greater at 1 day than at 3 days, although a more reliable distinguishing feature was the much greater density of extravasated plasma protein in the extracellular space at 1 day after the cold lesion. This finding is in keeping with the lower

Table $2 T_{2}$ relaxation times of each component, and the proportion of the total signal contributed by the long- $T_{2}$ component in normal and oedematous white matter

\begin{tabular}{llll}
\hline $\begin{array}{l}\text { Experimental } \\
\text { group }\end{array}$ & $\begin{array}{l}\text { Short- } T_{2} \\
(m s)\end{array}$ & $\begin{array}{l}\text { Long- } T_{2} \\
(m s)\end{array}$ & $\begin{array}{l}\% \\
\text { Long- } T_{2}\end{array}$ \\
\hline Control $(\mathrm{n}=4)$ & $67 \pm 4$ & $190 \pm 32$ & $21 \pm 7$ \\
l day cold $(\mathrm{n}=7)$ & $74 \pm 19$ & $337 \pm 60$ & $65 \pm 10$ \\
3 day cold $(\mathrm{n}=3)$ & $56 \pm 8$ & $285 \pm 109$ & $55 \pm 7$ \\
TET sulphate $(\mathrm{n}=5)$ & $70 \pm 13$ & $346 \pm 66$ & $24 \pm 4$ \\
\hline
\end{tabular}

protein content of oedematous white matter at 3 days, ${ }^{1112}$ which is the result of uptake and metabolism of the protein by astrocytes and vascular endothelial cells. ${ }^{13}$

The animals with cytotoxic oedema showed variable susceptibility to TET sulphate, and some modification of the dosage regime was required to produce comparable changes in the MRI appearances of the oedematous white matter. The experiments were terminated at 4 days since the pathological changes were well developed at this time and the clinical effects were not yet severe. Cytotoxic oedema was less severe than vasogenic in terms of the size of the ultrastructural oedema space and smaller increase in water content of the oedematous tissue. Furthermore, the protein content of the oedema fluid was different in that cytotoxic oedema fluid was unassociated with plasma protein in keeping with previous reports that the fluid resembles a plasma ultrafiltrate. ${ }^{5}$

MRI was sensitive in demonstrating both types of oedema, but on the basis of image appearance alone it was not possible to distinguish reliably between regions affected by cytotoxic and vasogenic oedema of similar severity. The anatomical extent of the image abnormalities corresponded with those seen on histological examination.

In vasogenic oedema, both SE and IR sequences clearly distinguished normal from abnormal tissue, but it was possible to differentiate between the area of the necrotic cortical lesion itself and the underlying oedema more easily on the SE images due to the greater sensitivity of these sequences to changes in $T_{2}$. It was also easier to appreciate regional variations in severity of the oedema on the SE images.

The $T_{1}$-dependant IR sequences were less useful in TET sulphate-induced oedema, first because increases in $T_{1}{ }^{\prime}$ were relatively small, and secondly because such increases only served to reduce the normal contrast between grey and white matter. Since the proportional increase in $T_{2}{ }^{\prime}$ was twice as great as in $T_{1}{ }^{\prime}$ in this type of oedema, the more $T_{2}$-dependent $S E$ sequences were most appropriate for demonstrating the extent of the pathological changes which could be seen most clearly when echo times greater than $120 \mathrm{~ms}$ were used. The correct choice of imaging sequences would obviously be important when examining a lesion with similar characteristics such as Reye's

Table 3 Water content of normal and oedematous white matter

\begin{tabular}{ll}
\hline Experimental group & Water content (\%) \\
\hline Control $(\mathrm{n}=4)$ & $65 \cdot 9 \pm 2$ \\
TET sulphate $(\mathrm{n}=3)$ & $72 \cdot 2 \pm 2 \cdot 9$ \\
Cold $(\mathrm{n}=3)$ & $78 \cdot 2 \pm 3 \cdot 6$ \\
\hline
\end{tabular}


syndrome $\mathrm{H}^{14}$ and the encephalopathy associated with mitochondrial myopathy. ${ }^{15}$

All experiments resulted in increases of both relaxation times, consistent with the findings of previous studies of these lesions by NMR spectroscopy. ${ }^{1617}$ The percentage increase in the relaxation times of the lesions ranged from $45-130 \%$, but the changes observed in individual lesions corresponded with the subjective assessment of the severity of the pathological changes in those case examined by electron microscopy. Go and Edzes ${ }^{16}$ have demonstrated that the severity of the oedema (as determined by the increase in tissue water content) is directly related to the resultant increase in $T_{1}{ }^{\prime}$ and $T_{2}{ }^{\prime}$, and it is likely that the range of increases in relaxation times found in the present experiments is a true reflection of the intrinsic variability of the oedematous process, and not the result of imprecise measurements.

There were significant differences in the pattern of increase in $T_{1}{ }^{\prime}$ and $T_{2}{ }^{\prime}$ between the experimental groups. In early vasogenic oedema in which the fluid was associated with large amounts of plasma protein, $\mathrm{T}_{1}{ }^{\prime}$ and $\mathrm{T}_{2}{ }^{\prime}$ increased in approximately the same proportion, whereas after 3 days, when much of the protein had been removed from the extracellular space, the proportional increase in $\mathrm{T}_{2}{ }^{\prime}$ was approximately $50 \%$ greater than that in $\mathrm{T}_{1}{ }^{\prime}$. Cytotoxic oedema, in which the oedema fluid was unassociated with protein, consistently showed an increase in $T_{2}{ }^{\prime}$ which was twice that in $T_{1}{ }^{\prime}$. The chemical nature of the oedema fluid thus appears to have a significant effect on the pattern of changes in $T_{1}{ }^{\prime}$ and $T_{2}{ }^{\prime}$. As the plasma protein is removed from the expanded extracellular space over a period of days following the cold lesion, so the pattern of change in the relaxation times approaches that seen in cytotoxic oedema in which the oedema fluid in the vacuoles is unassociated with protein.

The pattern of elevation of the relaxation times was independent of the severity of the oedema in both models; over a wide range of severity the pattern remained consistent within each experimental group. Two individual cold lesions which increased in severity between 1 and 3 days still conformed to the expected pattern of changes in the relaxation times outlined above.

These findings may be explicable in terms of what is known about the relaxation behaviour of protein solutions. ${ }^{18}$ Protein molecules increase the relaxation efficiency of water protons, shortening both $T_{1}$ and $T_{2}$ as a result of restriction of the free motion of the water. When protein is associated with water at the concentration found in plasma, $T_{2}$ is much shorter than $T_{1}$, but at progressively lower concentrations $T_{2}$ increases more rapidly than $T_{1}$ until they become equal for pure water. The oedema fluid in 1 day vasogenic, 3 day vasogenic, and cytotoxic oedema is asso- ciated with protein at high, intermediate, and zero concentrations respectively, and therefore it might be expected that 1 day vasogenic oedema would result in the smallest increase in $T_{2}{ }^{\prime}$ relative to $T_{1}{ }^{\prime}$, and cytotoxic oedema the largest, as we have observed.

There are potential difficulties in the interpretation of relaxation data calculated from only two sequences. As noted by Brandt-Zawadzki et al, ${ }^{19}$ variation of the sequence repetition time results in variations in the calculated relaxation times. In our experiments, the sequence parameters were kept constant, and the low variance of the relaxation data from the control animals $\left(\mathrm{T}_{1}{ }^{\prime}=476 \pm 10 ; \mathrm{T}_{2}{ }^{\prime}=81\right.$ \pm 4 ) suggests that our calculated values were sufficiently precise.

As previously mentioned, there is a further problem with relaxation time measurement using a two-point method. When tissue magnetisation decays in a nonexponential fashion, calculation of $T_{1}{ }^{\prime}$ and $T_{2}{ }^{\prime}$ by such a method will inevitably produce data that are not fully descriptive of the relaxation behaviour of the tissue. While recognising this limitation, these experiments have shown that they can provide an insight into the nature of the fluid in cerebral oedema, and might be usefully applied to the assessment of abnormal permeability of the blood-brain barrier and its therapeutic modification in man.

Further information about the microscopic environment of tissue water can be obtained by examining its magnetisation decay characteristics. The $T_{1}$ decays were always monoexponential in these experiments. Previous workers have been unable to detect more than one $T_{1}$ component in vasogenic oedema, ${ }^{1720}$ although Go and Edzes ${ }^{16}$ reported a biexponential $T_{1}$ decay when studying cytotoxic oedema in rats by in vitro spectroscopy after large, single intravenous doses of TET. It is uncertain to what extent such in vitro findings can be extrapolated to in vivo tissue relaxation behaviour in view of the inevitable alternations in the physical properties of tissues which occur at death.

We have found the $T_{2}$ decays to be biexponential in both normal and oedematous white matter, although it is probable that there are further, smaller components of tissue water which have very short $T_{2}$ relaxation times of the order of microseconds or a few milliseconds. ${ }^{21}$ There were no significant differences in the relaxation times of the short- $T_{2}$ components between any of the groups, and in keeping with the relatively unaltered ultrastructural appearance of the intracellular space in these lesions, it is reasonable to ascribe this component to intracellular water which is unable to exchange with either the true extracellular space or water within intramyelinic vacuoles rapidly enough to yield as a single relaxation component.

The relative amplitude of the long- $T_{2}$ component 
of normal white matter, representing extracellular water, was $21 \%$, which is similar to the size of the in vivo extracellular space in this tissue estimated by other methods. ${ }^{22} 23$ The relative amplitude of this component may therefore provide a means of determining the degree of expansion of the extracellular space in vasogenic oedema. In these experiments, the mean relative amplitude in 1 day vasogenic oedema was $65 \%$, reaching $76 \%$ in the most severe individual lesion. These values were compatible with the size of the expanded extracellular space which we observed electron microscopically.

In cytotoxic oedema the relaxation time of the long- $T_{2}$ component, representing water within the vacuoles $(346 \mathrm{~ms})$ was considerably greater than that of water in the normal extracellular space $(190 \mathrm{~ms})$, but we were unable to detect separate $T_{2}$ components for extracellular and vacuolar water. This may have been due to compression of the extracellular space by the expanding intramyelinic vacuoles, reducing the size of its signal beyond the sensitivity of our technique.

NMR spectroscopy has been used for some time to study the physico-chemical environment of tissue water in vitro, and we have shown that it is possible to obtain similar information in vivo using quantitative imaging techniques. We have been able to demonstrate that while the anatomical extent of a pathological process can be accurately delineated by NMR images, consideration of the quantitative NMR characteristics of the abnormal tissue can provide further insight into the nature of the underlying changes.

The application of these principles to patients has permitted a distinction to be made between acute brainstem lesions of the kind with which multiple sclerosis can present, and the chronic brainstem lesions of the established disease. ${ }^{24}$ The further development of this approach has obvious implications for early diagnosis and the monitoring of therapy, and may facilitate the distinction between different classes of pathological process by NMR imaging.

We are grateful to the Cerebral Oedema Research Group for carrying out the measurements of water content, and to Miss Jenny Small for expert technical assistance with the morphological studies and illustrations. The work was carried out on the MRI facility provided by the Multiple Sclerosis Society, and was supported by a grant from the MRC.

\section{References}

1 Ormerod IEC, Douboulay EGH, McDonald WI. Imaging and Multiple Sclerosis. In: McDonald WI, Silverberg DH, eds. Multiple Sclerosis. London: Butterworths, 1986.

2 Mathur-De Vre $R$. Biochemical implications of the relaxation behaviour of water related to NMR imaging. Br J Radiol 1984;57:955-76.

3 Gazendam J, Go GK, van Zanten AK. Composition of isolated edema fluid in cold-induced brain edema. $J$ Neurosurg 1979;51:70-7.

4 Lee JC, Bakay L. Ultrastructural changes in the edematous central nervous system. Arch Neurol 1965; 13:48-58.

5 Bakay L. Morphological and chemical studies in cerebral oedema: Triethyltin-induced oedema. J Neurol Sci 1965;2:52-67.

6 Barnes D, McDonald WI, Tofts PS, Johnson G, Landon DN. Nuclear magnetic resonance imaging in experimental cerebral oedema. $J$ Neurol Neurosurg Psychiatry 1986;49:1341.

7 Young IR, Bailes DR, Burl M, et al. Initial clinical evaluation of a whole body nuclear magnetic resonance tomograph. J Comput Assist Tomog 1982;6(1):1-18.

8 Go GK, Ebels EJ, Beks JWF, Ter Weeme CA. The spreading of cerebral edema from a cold injury in cats. Psychiatry Neurol Neurochir 1967;70:403-11.

9 Lee JC, Bakay L. Ultrastructural changes in the edematous central nervous system. Arch Neurol 1966;14:36-49.

10 Long DM, Maxwell RE, French LA. The effects of glucosteroids upon cold induced brain edema. J Neuropathol Exp Neurol 1971;30:680-97.

11 Cutler RWP, Watters GV, Barlow CF. I $^{125}$-labelled protein in experimental brain edema. Arch Neurol 1964;11:225-38.

12 Rasmussen LE, Klatzo I. Protein and enzyme changes in cold injury edema. Acta Neuropathol (Berlin) 1969;13:12-28.

13 Crockard HA. Brain swelling, brain oedema and the blood-brain barrier. In: Crockard A, Hayward R, Hoff JT, eds. Neurosurgery. The scientific basis of clinical practice. Oxford: Blackwell Scientific, 1985.

14 Partin JC, Partin JS, Schubert WK, McLaurin RL. Brain ultrastructure in Reye's syndrome. $J$ Neuropathol Exp Neurol 1975;34:425-44.

15 DiMauro S, Bonilla E, Zeviani M, et al. Mitochondrial myopathies. Ann Neurol 1985;17:521-38.

16 Go GK, Edzes HT. Water in brain edema. Arch Neurol 1975;32:462-5.

17 Naruse S, Horikawa Y, Tanaka C, et al. Nuclear magnetic resonance studies on brain edema. $J$ Neurosurg 1982;56:747-52.

18 Daszkiewicz OK, Hennel JW, Lubas B. Protein magnetic relaxation and protein hydration. Nature 1963;200: 1006-7.

19 Brandt-Zawadzki M, Bartkowski HM, Ortendahl DA, et al. Am J Neuroradiol 1984;5:125-9.

20 Bakay L, Kurland RJ, Parrish RG, et al. Nuclear magnetic resonance studies in normal and edematous brain tissue. Exp Brain Res 1975;23:241-8.

21 Foster KR, Resing HA, Garroway AN. Bounds on "Bound water": transverse nuclear magnetic relaxation in barnacle muscle. Science 1976;194:324-6.

22 Rees S, Cragg BG, Everitt AV. Comparison of extracellular space in the mature and ageing rat brain using a new technique. J Neurol Sci 1982;53:347-57.

23 Van Harreveld $A$. The extracellular space in the vertebrate central nervous system. In: Bourne $\mathbf{G H}$, ed. The Structure and Function of Nevous Tissue. Vol 4. New York and London: Academic Press, 1972.

24 Ormerod IEC, Bronstein A, Rudge P, et al. Nuclear magnetic resonance imaging in clinically isolated lesions of the brainstem. $J$ Neurol Neurosurg Psychiatry 1987 (in press). 